\title{
Near-infrared spectroscopy and plasma homovanillic acid levels in bipolar disorder: a case report
}

This article was published in the following Dove Press journal:

Neuropsychiatric Disease and Treatment

25 March 2014

Number of times this article has been viewed

\author{
Itaru Miura ${ }^{1,2}$ \\ Soichi Kono' \\ Sachie Oshima' \\ Keiko Kanno-Nozaki' \\ Hirobumi Mashiko' \\ Shin-Ichi Niwa' \\ Hirooki Yabe' \\ 'Department of Neuropsychiatry, \\ School of Medicine, Fukushima \\ Medical University, Fukushima, Japan; \\ ${ }^{2}$ Division of Psychiatry Research, The \\ Zucker Hillside Hospital, Glen Oaks, \\ NY, USA
}

\begin{abstract}
Misdiagnosis of bipolar disorder is a serious, but not unusual problem for patients. Nevertheless, there are few biomarkers for distinguishing unipolar and bipolar disorder. Near-infrared spectroscopy (NIRS) is a noninvasive and useful method for the measurement of hemoglobin concentration changes in the cortical surface area, which enables the assessment of brain function. We measured NIRS and plasma monoamine metabolite levels in a patient with bipolar disorder. A 22-year-old man was admitted due to major depression. At admission, NIRS findings showed oxygenated hemoglobin reincrease in the posttask period, which is characteristic of schizophrenia. After treatment with paroxetine, he became manic with psychotic symptoms. His plasma level of homovanillic acid just before the manic switch was ten times higher than that just after paroxetine initiation. Treatment with lithium and antipsychotics was successful, and plasma homovanillic acid decreased after treatment. In this case, the NIRS findings may predict a possible risk of a manic switch, which is likely induced by paroxetine. NIRS may be able to help distinguish unipolar and bipolar disorder in clinical settings.
\end{abstract}

Keywords: near-infrared spectroscopy, bipolar disorder, homovanillic acid, diagnosis, biomarker

\section{Introduction}

Misdiagnosis of bipolar disorder is a potentially serious problem for patients, which sometimes leads to inappropriate treatment. ${ }^{1}$ However, it is not unusual for bipolar disorder to be misdiagnosed as a major depressive disorder. ${ }^{2}$ Previous studies have shown that the proportion of cases of bipolar disorder misdiagnosed as a major depressive disorder is $20.8 \%-40 \% .^{2-4}$ Nevertheless, there are few biomarkers for accurate diagnosis. If patients become depressive for the first time and have never experienced a manic episode, it is difficult to determine whether that depressive episode is unipolar or bipolar depression, and the risk of a manic switch is unknown. Near-infrared spectroscopy (NIRS) is a noninvasive and useful method that uses near-infrared light to measure hemoglobin concentration changes in the cortical surface area. Although changes in the NIRS signals measured on the forehead during the word fluency task may be partially associated with changes in skin blood flow, ${ }^{5}$ the changes in oxygenated hemoglobin ([oxyHb]) are associated with changes in the regional cerebral blood volume. NIRS enables the assessment of brain function, and recent studies have shown that the frontal hemodynamic patterns detected by NIRS can be a biomarker for the diagnosis of major psychiatric disorders. ${ }^{6,7}$

In the present case, NIRS during the word fluency task was measured during the clinical course of bipolar disorder. In brief, [oxyHb] and deoxygenated hemoglobin 
([deoxyHb]) were measured with a 52-channel NIRS machine (Hitachi ETG-4000; Hitachi Medical Corporation, Tokyo, Japan) at two wavelengths of near-infrared light (695 and $830 \mathrm{~nm}$ ), the absorption of which was measured, and [oxyHb] and [deoxyHb] were calculated as previously described. ${ }^{8}$ The distance between pairs of sourcedetector probes was set at $3.0 \mathrm{~cm}$ and each measured area between pairs of source detector probes was defined as a "channel". The probes of the NIRS machine were fixed with $3 \times 11$ thermoplastic shells, with the lowest probes located along the Fp1-Fp2 line according to the international 10-20 system used in electroencephalography. We used the word fluency task because it is easy to understand and execute, and it is also an executive task that produces distinct differences in performance and neuroimaging data among each diagnostic group of psychiatric disorders. ${ }^{7}$ Additionally, plasma levels of homovanillic acid (HVA), a dopamine metabolite, and 3-methoxy-4hydroxyphenylglycol (MHPG), a noradrenaline metabolite, were measured longitudinally using high performance liquid chromatography. In our hospital, we measured both plasma levels of monoamine metabolites (every 3 weeks in principle) and NIRS (before and after the treatment) in psychiatric inpatients with mood disorders to investigate the association between plasma monoamine metabolites and NIRS signals. This protocol was approved by the ethics committee of Fukushima Medical University, and the patient consented to participate after having been informed of the purpose of the study.

\section{Case report}

The patient was a 22-year-old man who had no history of psychiatric illness. He was admitted to our hospital due to major depression that was diagnosed according to the Diagnostic and Statistical Manual for Mental Disorders, Fourth Edition, with moderate sadness, agitation, retardation, and difficulty in concentrating. The clinical course after admission is summarized in Figure 1. We treated the patient with paroxetine and lorazepam for about 4 weeks, but treatment was not successful. On day 4, his Montgomery Åsberg Depression Rating Scale (MADRS) score was 44, and his plasma levels of HVA and MHPG were $4.6 \mathrm{ng} / \mathrm{mL}$ and $1.9 \mathrm{ng} / \mathrm{mL}$, respectively. Figure 2 shows the waveform

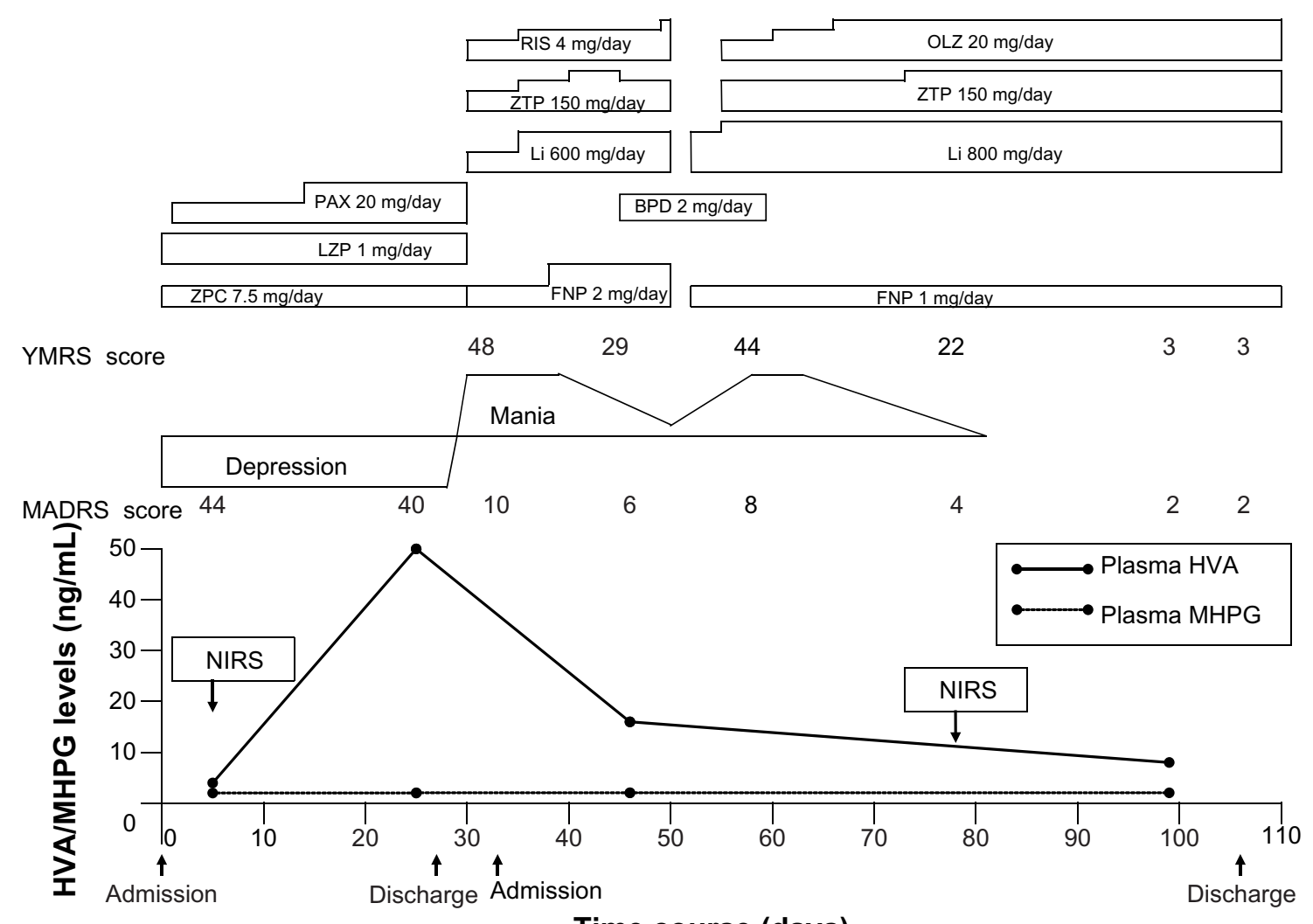

Time course (days)

Figure I Clinical course after admission.

Note: Figures next to each drug mean maximal dose.

Abbreviations: BPD, biperiden; FNP, flunitrazepam; HVA, homovanillic acid; Li, lithium carbonate; LZP, lorazepam; MADRS, Montgomery Åsberg Depression Rating Scale; MHPG, 3-methoxy-4hydroxyphenylglycol; NIRS, near-infrared spectroscopy; OLZ, olanzapine; PAX, paroxetine; RIS, risperidone; YMRS, Young Mania Rating Scale; ZPC, zopiclone; ZTP, zotepine. 


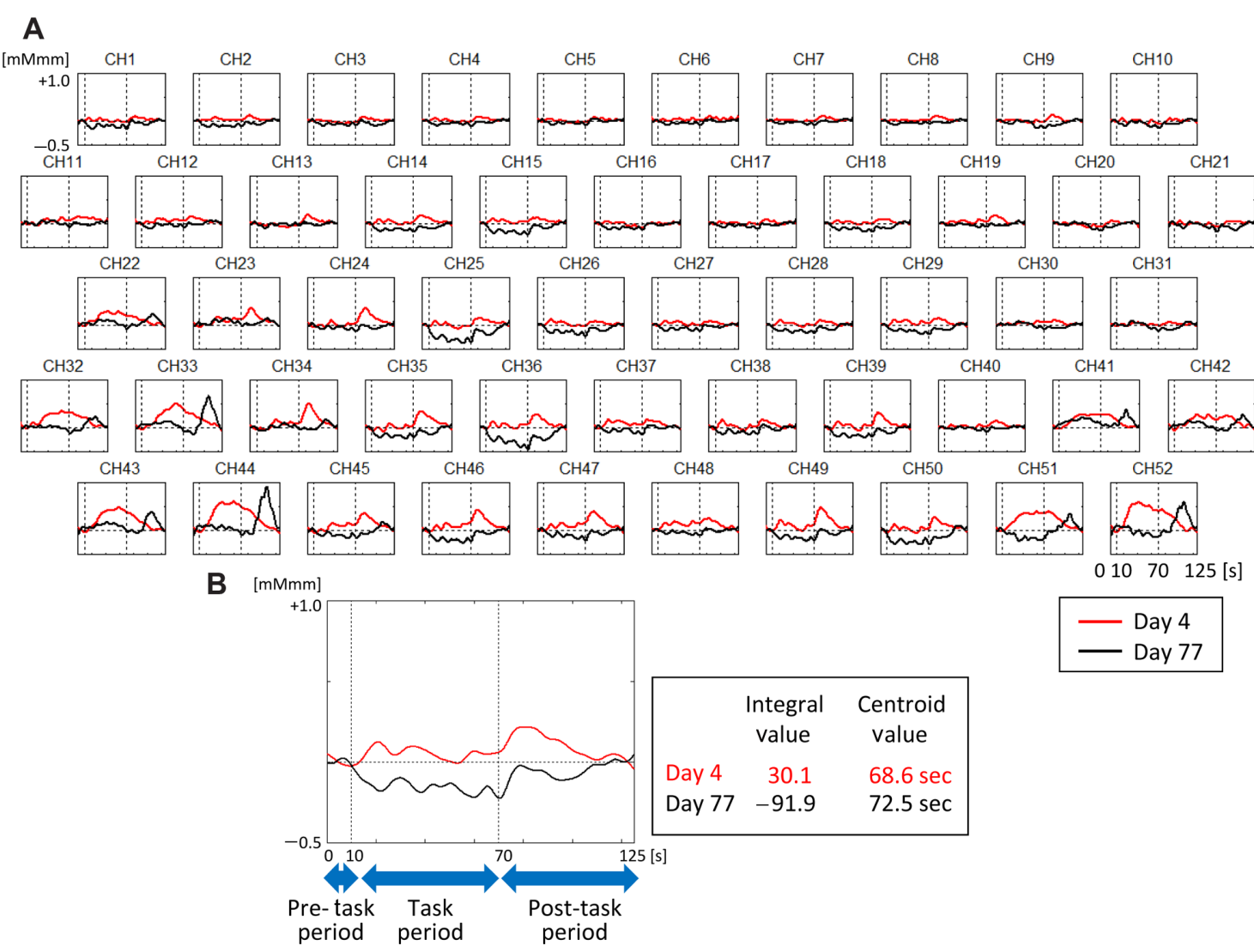

Figure 2 Waveforms of oxygenated hemoglobin.

Notes: (A) Waveforms of oxygenated hemoglobin during the cognitive activation of the word fluency task. (B) Grand average waveforms of oxygenated hemoglobin in the frontal region ( $\mathrm{CH} 25,26,27,28,36,37,38,46,47,48$, and 49).

of [oxyHb] during the cognitive activation of the word fluency task. NIRS signal changes were measured during a 10 second pretask baseline period, a 60 second task period, and a 55 second posttask baseline period. The initial syllables assigned were changed every 20 seconds $(/ \mathrm{a} /, / \mathrm{ka} /$, and $/ \mathrm{sa} /$, respectively) during the 60 second task period. During the task period, the patient was instructed to generate as many Japanese words beginning with the designated syllable as possible. In the pretask and the posttask baseline periods, the patient was instructed to repeat a train of syllables
$(/ \mathrm{a} /, / \mathrm{i} /, / \mathrm{u} /, \mathrm{e} /$, and $/ \mathrm{o} /)$. He sat on a chair in a room with daylight with his eyes open throughout the measurements. We focused on increases in [oxyHb], because this appears to reflect task-related cortical activation more directly than decreases in [deoxyHb]. ${ }^{7}$ We defined the patterns of NIRS waveforms using the grand average waveforms in the frontal region ( $\mathrm{CH} 25-28, \mathrm{CH} 36-38$, and $\mathrm{CH} 46-49$; Figure 3 ) and two simple visual indices, ${ }^{7}$ integral value, and centroid value. The integral value describes the size of the hemodynamic response during the 60 second activation task period, whereas

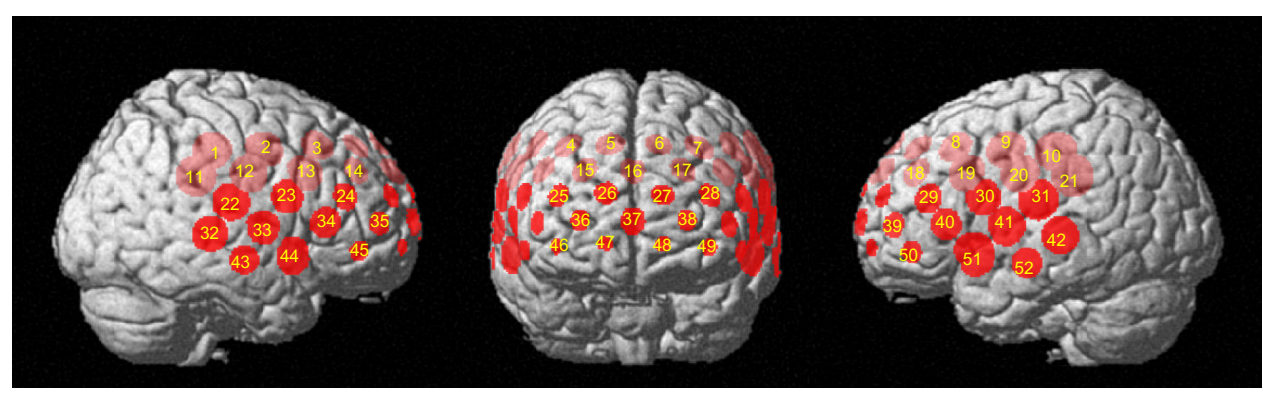

Figure 3 Regions of the near-infrared spectroscopy signals.

Notes: The location of near-infrared spectroscopy measurements were anatomically labeled in the standard brain space. Reprinted from Neurolmage, Volume 85 , Takizawa R, Fukuda M, Kawasaki S, et al; Joint Project for Psychiatric Application of Near-Infrared Spectroscopy (JPSY-NIRS) Group. Neuroimaging-aided differential diagnosis of the depressive state, pages 498-507, Copyright (2014), with permission from Elsevier.? 
the centroid value serves as an index of time course changes throughout the task, with periods representing the timing of the hemodynamic response. ${ }^{7}$ A recent study reported that the optimal threshold of the centroid value of the frontal region between major depressive disorder and two other disorders (schizophrenia and bipolar disorder) is 54 seconds (major depressive disorder $<$ other 2 disorders). ${ }^{7}$ On day 4 , the centroid value of the grand average in the central region was 68.6 seconds (Figure 2), and the NIRS waveforms showed an [oxyHb] reincrease in the posttask period ( $\mathrm{CH} \mathrm{34,} \mathrm{35,} \mathrm{36,} \mathrm{39,} \mathrm{45,} \mathrm{46,} \mathrm{47,} \mathrm{and} \mathrm{49;} \mathrm{Figure} \mathrm{2),} \mathrm{which} \mathrm{is}$ characteristic of schizophrenia. ${ }^{6,79-11}$ On day 25, his MADRS score was 40, his plasma HVA level was markedly higher at $49.3 \mathrm{ng} / \mathrm{mL}$, and his plasma MHPG level was $1.6 \mathrm{ng} / \mathrm{mL}$. On day 27 , he was voluntarily discharged from the hospital, but was still in a depressive state. On day 30, 3 days after leaving the hospital, he became manic and was readmitted to our hospital. He was in a state of psychotic mania with elevated mood, grandiosity, aggression, auditory hallucination, and thought broadcast, with a Young Mania Rating Scale (YMRS) score of 48. Paroxetine and lorazepam were discontinued. We subsequently treated him with lithium, risperidone, and zotepine for approximately 3 weeks, and his manic state improved. On day 46, his YMRS score was 29, and plasma levels of HVA and MHPG were $17 \mathrm{ng} / \mathrm{mL}$ and $1.3 \mathrm{ng} / \mathrm{mL}$, respectively. However, he refused drugs because of extrapyramidal symptoms and became manic with psychotic symptoms again. Therefore, we tried a combination of lithium, olanzapine, and zotepine, and the treatment was successful. On day 77, the NIRS waveforms turned to a negative pattern (Figure 2), which was defined by an integral value of the grand average in the central region $<0$, whereas there were [oxyHb] reincreases in the posttask period ( $\mathrm{CH} \mathrm{33,} \mathrm{41,} \mathrm{42,} \mathrm{43,} \mathrm{44,} \mathrm{51,} \mathrm{and} \mathrm{52).} \mathrm{On} \mathrm{day} \mathrm{99,} \mathrm{his}$ YMRS score decreased to 3, and his plasma levels of HVA and MHPG were $8.7 \mathrm{ng} / \mathrm{mL}$ and $1.9 \mathrm{ng} / \mathrm{mL}$, respectively. He was discharged from the hospital on day 106 . Nine months later, he was off medication, became manic again, and was admitted to another psychiatric hospital.

\section{Discussion}

To the best of our knowledge, this is the first case report to measure both NIRS and plasma levels of monoamine metabolites in a patient with bipolar disorder, although the clinical course in this patient was not usual as the mania occurred within the first weeks of the initiation of serotonin selective reuptake inhibitor (SSRI) treatment. In this case, it was difficult to diagnose bipolar depression from only clini- cal symptoms at his first admission. We could not predict the subsequent manic switch with psychotic features, which was likely caused by paroxetine. However, the results of NIRS on day 4 showed a pattern of schizophrenia, characterized by a smaller [oxyHb] increase during the first half of the task and an [oxyHb] reincrease in the posttask period, ${ }^{4,9-11}$ but not a unipolar depression pattern, which is characterized by only a smaller [oxyHb] increase during the first half of the period. ${ }^{4,9,12,13}$ Care must be taken to interpret these findings as patterns of diseases because of the small number of studies. $\mathrm{An}[\mathrm{oxyHb}]$ reincrease in the posttask period is considered as inefficient activation of the prefrontal cortex, ${ }^{4,9,11}$ which is one of the quantitative abnormalities in schizophrenia. ${ }^{14}$ These results may have predicted a possible risk of a manic switch in this patient. A recent study ${ }^{7}$ reported that the frontal hemodynamic patterns detected by NIRS accurately distinguished between patients with major depressive disorder and those with bipolar disorder or schizophrenia with depressive symptoms. This is consistent with the results in this patient. Although the clinical status of bipolar depression may be different from that of unipolar depression, ${ }^{1}$ it is not easy to clearly distinguish without a previous manic episode. In fact, most bipolar disorder patients with depressive symptoms are initially diagnosed as having major depression. ${ }^{2}$ Because there are few biomarkers for diagnosis, NIRS findings may help for distinguishing unipolar and bipolar disorders. However, NIRS signals changed after treatment with antipsychotics and lithium in this patient. Although it is believed that NIRS waveforms are a possible trait marker, NIRS signals may change with the state or the medication.

Interestingly, NIRS findings in this case showed a pattern of schizophrenia, not a pattern of bipolar disorder, which is characterized by a smaller [oxyHb] increase during the early period, and larger [oxyHb] increase during the late period of this task. ${ }^{15}$ This result is consistent with a manic episode in this case that included psychotic symptoms such as hallucination and thought broadcast. Furthermore, 5 days before the manic switch, the patient's plasma HVA level was almost ten times higher than just after admission. Because the HVA level change occurred in tandem with the manic symptoms during the clinical course, it is estimated that the manic episode in this case was associated with dopaminergic function. Plasma HVA levels have been considered as a possible indicator in the treatment of schizophrenia ${ }^{16,17}$ and bipolar disorder. ${ }^{18,19}$ Furthermore, because SSRIs act on dopaminergic and serotonergic neural systems, ${ }^{20}$ the increase in the plasma HVA level in this patient may have been induced by paroxetine. 
A previous study reported that psychosis or mania induced by SSRIs showed a pattern of increased plasma HVA levels. ${ }^{21}$ This is consistent with our findings of the changes in plasma HVA levels in this patient. Taken together, the NIRS findings in this patient may be based on dopaminergic dysfunction. However, the changes in the NIRS may not be directly associated with the changes in plasma HVA levels, because NIRS findings are based on frontal brain functions, whereas plasma HVA levels are related to psychotic symptoms, ${ }^{22}$ which seem to be a reflection of the mesolimbic dopamine system.

\section{Conclusion}

In this case, NIRS findings in a depressive state may predict the possible risk for a manic switch. NIRS may be able to help distinguish unipolar and bipolar disorders in clinical settings. Longitudinal monitoring of plasma HVA levels may be useful in the treatment of bipolar disorder, in particular in patients with psychotic symptoms like this case. These possible biomarkers may contribute to accurate diagnosis and appropriate treatment for bipolar disorder.

\section{Disclosure}

The authors report no conflicts of interest in this work.

\section{References}

1. Ghaemi SN, Ko JY, Goodwin FK. "Cade's disease" and beyond: misdiagnosis, antidepressant use, and a proposed definition for bipolar spectrum disorder. Can J Psychiatry. 2002;47:125-134.

2. Ghaemi SN, Boiman EE, Goodwin FK. Diagnosing bipolar disorder and the effect of antidepressants: a naturalistic study. J Clin Psychiatry. 2000;61:804-808.

3. Xiang YT, Zhang L, Wang G, et al. Sociodemographic and clinical features of bipolar disorder patients misdiagnosed with major depressive disorder in China. Bipolar Disord. 2013;15:199-205.

4. Ghaemi SN, Sachs GS, Chiou AM, Pandurangi AK, Goodwin K. Is bipolar disorder still underdiagnosed? Are antidepressants overutilized? $J$ Affect Disord. 1999;52:135-144.

5. Takahashi T, Takikawa Y, Kawagoe R, Shibuya S, Iwano T, Kitazawa S. Influence of skin blood flow on near-infrared spectroscopy signals measured on the forehead during a verbal fluency task. Neuroimage. 2011;57:991-1002.

6. Suto T, Fukuda M, Ito M, Uehara T, Mikuni M. Multichannel nearinfrared spectroscopy in depression and schizophrenia: cognitive brain activation study. Biol Psychiatry. 2004;55:501-511.
7. Takizawa R, Fukuda M, Kawasaki S, et al; Joint Project for Psychiatric Application of Near-Infrared Spectroscopy (JPSY-NIRS) Group. Neuroimaging-aided differential diagnosis of the depressive state. Neuroimage. 2014;85:498-507.

8. Maki A, Yamashita Y, Ito Y, Watanabe E, Mayanagi Y, Koizumi H. Spatial and temporal analysis of human motor activity using noninvasive NIR topography. Med Phys. 1995;22:1997-2005.

9. Kinou M, Takizawa R, Marumo K, et al. Differential spatiotemporal characteristics of the prefrontal hemodynamic response and their association with functional impairment in schizophrenia and major depression. Schizophr Res. 2013;150:459-467.

10. Shimodera S, Imai Y, Kamimura N, et al. Mapping hypofrontality during letter fluency task in schizophrenia: a multi-channel near-infrared spectroscopy study. Schizophr Res. 2012;136:63-69.

11. Takizawa R, Kasai K, Kawakubo Y, et al. Reduced frontopolar activation during verbal fluency task in schizophrenia: a multi-channel nearinfrared spectroscopy study. Schizophr Res. 2008;99:250-262.

12. $\mathrm{Pu} \mathrm{S}$, Nakagome K, Yamada T, et al. The relationship between the prefrontal activation during a verbal fluency task and stress-coping style in major depressive disorder: a near-infrared spectroscopy study. J Psychiatr Res. 201;46:1427-1434.

13. Noda T, Yoshida S, Matsuda T, et al. Frontal and right temporal activations correlate negatively with depression severity during verbal fluency task: a multi-channel near-infrared spectroscopy study. J Psychiatr Res. 2012;46:905-912.

14. Meyer-Lindenberg A. From maps to mechanisms through neuroimaging of schizophrenia. Nature. 2010;468:194-202.

15. Kameyama M, Fukuda M, Yamagishi Y, et al. Frontal lobe function in bipolar disorder: A multichannel near-infrared spectroscopy study. Neuroimage. 2006;29:172-184.

16. Kelley ME, Yao JK, van Kammen DP. Plasma catecholamine metabolites as markers for psychosis and antipsychotic response in schizophrenia. Neuropsychopharmacology. 1999;20:603-611.

17. Miura I, Takeuchi S, Katsumi A, et al. Effects of aripiprazole and the Taq1A polymorphism in the dopamine D2 receptor gene on the clinical response and plasma monoamine metabolites level during the acute phase of schizophrenia. J Clin Psychopharmacol. 2012;32:106-109.

18. Post RM, Jimerson DC, Bunney WE Jr, Goodwin FK. Dopamine and mania: behavioral and biochemical effects of the dopamine receptor blocker pimozide. Psychopharmacology (Berl). 1980;67:297-305.

19. Mazure CM, Bowers MB. Pretreatmnent plasma HVA predicts neuroleptic response in manic psychosis. J Affect Disord. 1998;48:83-86.

20. Willner P, Hale AS, Argyropoulos S. Dopaminergic mechanism of antidepressant action in depressed patients. J Affect Disord. 2005;86: $37-45$.

21. Fortunati F, Mazure C, Preda A, Wahl R, Bowers M Jr. Plasma catecholamine metabolites in antidepressant-exacerbated mania and psychosis. J Affect Disord. 2002;68:331-334.

22. Yoshimura R, Nakano Y, Hori H, Ikenouchi A, Ueda N, Nakamura J. Effect of risperidone on plasma catecholamine metabolites and brainderived neurotrophic factor in patients with bipolar disorders. Hum Psychopharmacol Clin Exp. 2006;21:433-438.
Neuropsychiatric Disease and Treatment

\section{Publish your work in this journal}

Neuropsychiatric Disease and Treatment is an international, peerreviewed journal of clinical therapeutics and pharmacology focusing on concise rapid reporting of clinical or pre-clinical studies on a range of neuropsychiatric and neurological disorders. This journa is indexed on PubMed Central, the 'PsycINFO' database and CAS

\section{Dovepress}

The manuscript management system is completely online and includes a very quick and fair peer-review system, which is all easy to use. Visit http://www.dovepress.com/testimonials.php to read real quotes from published authors. 